\title{
MASS DEFINITION, MASS MEASUREMENT AND RECOMMENDATIONS
}

\author{
David W. Braudaway \\ Sandia National Laboratories \\ Division 7342
}

Albuquerque, NM 87185
SAND- $-90-1510$

DE9 1000458

\begin{abstract}
Mass measurements may be greatly entanced by an understanding of the operation of balances, the effects of buoyancy, practices encountered in weighing and the impacts and meanings of revelant mass standards. Beginning with the basic forces acting on weights, the equations brought to equality in balances are developed. These give explanation of the weighing process and an appreciation for some balance characteristics. The requirements of relevant mass standards are also reviewed. Recommendations are made for operation of practical mass. calibration. An appendix is attached which gives computation examples using "apparent mass" and also gives a method for determining the density of mass artifacts or unknown materials.
\end{abstract}

\section{A. BACKGROUND}

Problems are regularly experienced in measurement of mass. Such problems might we 11 be expected as many balances resolve mass to a part in a million or better. Investigation has shown that these problems reside primarily in limitations on understanding of the equipment, the behavior of both equipment and mass standards and/or the meaning of values assigned to mass standards. The limitations are made more severe by limited availability of information on mass behavior and measurement. The difficulties arise as a result of one or more of the following factors:

1. A general lack of appreciation for and understanding of the importance of correction for the buoyant effect of air.

2. Changes in the reference density, the conditions under which "apparent mass" is determined, and the method of designating laboratory weights and precision mass standards.

3. The marketing of new balances, many with electronic readout, which have characteristics differing from those previously available.

The problems have been seen at the Sandia National Laboratories Primary Standards Laboratory (PSL) as a request for more accurate weights or for better weights. The magnitude of the uncertainty assigned to weights is currently at the practical 1 imit and cannot realistically be decreased either by improved technique or different equipment. For this reason and to enhance understanding of both mass standards and balances, the following discussion is offered. 
Page 2

Examples of calculation and the methods of calculation are included in the Appendix.

\section{B. BUOYANCY AND ITS EFFECTS}

Archimedes' Principle states that "any object immersed in a fluid will suffer an apparent loss of weight equal to the weight of the same volume of the fluid itself". In terms common to weighing, this principle states that the apparent mass of an object immersed in a fluid is smaller than its true mass (mass in vacuum) by the mass of the fluid displaced. The volume of the object and the volume of the displaced fluid are the same and the density (mass per unit volume) is the factor that changes each to mass.

When one thinks of a fluid, the first thought is of something like water, but air is also a fluid and Archimedes' Principle applies. In order to fully analyze buoyancy and its effects, three separate topics must be considered; force, weighing or mass comparison, and "apparent mass."

\section{TOPIC 1. FORCE.}

Force is described by the equation:

$$
\mathrm{f}=\mathrm{ma} \quad \text { or } \quad \mathrm{f}=\mathrm{mg} \text {; }
$$

where $f$ is the force, $m$ is the true mass (mass in vacuum), a is acceleration, and $\mathrm{g}$ is acceleration due to gravity. A further complication arises when the English system is used because both mass and force have the unit Pounds. For the English system, $g$, the acceleration due to gravity, is replaced by the ratio of the local acceleration due to gravity to the reference acceleration due to gravity. The force effect is more easily understood in Metric units because the unit of mass is the Kilogram and the unit of force is the Newton. With either system of units, knowledge of the local constant for acceleration due to gravity is required; in the English system the reference gravity constant must also be known.

For a weight used in a medium other than vacuum, the buoyant effect of Archimedes' Principle produces a force acting on the mass which is in the opposite direction to that produced by gravity. The magnitude of this force is:

$$
\mathrm{f}=\mathrm{v} \rho \mathrm{g}=\mathrm{m} \rho \mathrm{g} / \mathrm{d}
$$

where $f$ is the force, $v$ is the volume displaced, $g$ is the acceleration due to gravity, $m$ is the true mass (in vacuum), $\rho$ is the density of the buoyant medium, and $d$ is the density of the weight. Note that $m, d$, and $v$ are all properties of the weight and that $\rho / d$ is the ratio of fluid density to the density of the weight. Note also that the net force on the weight i.s : 


$$
\mathrm{f}=\mathrm{mg}-\mathrm{mg} \rho / \mathrm{d}=\mathrm{mg}(1-\rho / \mathrm{d}) .
$$

The term $(1-\rho / d)$ is the fraction of the force left after the buoyant correction; $\rho / d$ gives the defect or fraction lost. The following table 1 ists the magnitudes of the defect in air at two altitudes (for weights with density 7.9 grams/cc):

TABLE I

$\begin{array}{lll}\begin{array}{l}\text { ALTITUDE } \\ \text { (feet) }\end{array} & \begin{array}{l}\text { AIR DENSITY } \\ \text { (nilligrams/cc) }\end{array} & \begin{array}{l}\text { DEFECT } \\ \text { (parts per } \\ \text { million) }\end{array} \\ 0 \text { (sea level) } & 1.2 & 151.9 \\ 5280 \text { (1 mile) } & 0.986 & 124.8\end{array}$

Not only are the magnitudes of the two values of defect relatively large, but the difference between the two is quite significant even when the uncertainty of mass value (true mass) is $10 \mathrm{ppm}$. For this reason, great care must be taken in determining air or fluid density accurately when using dead weight to produce force or when using weights on a suitable piston to produce pressure (force per unit area). When combinations of weights are used having different densities, it is necessary to compute the force for each and to sum the value to get the total. The sum is represented by:

$$
\left.\sum_{i=1}^{n} m(i) g i 1-\rho / d(i)\right]
$$

where $m(i)$ is the true mass of weight $i$ of the set which has density $d(i)$, $g$ is the acceleration due to gravity, and $\rho$ is density of the air or fluid. This calculation is made much easier if all the weights have the same density in which case only the masses are summed and the buoyant fraction appears in a common multiplier.

\section{TOPIC 2. WEIGHING OR MASS COMPARISON}

The process of weighing involves comparison of the force on a set of reference weights to the force on an unknown. Buoyancy is important as each weight of the reference set and the unknown will generally have lifferent densities. Also important is the constancy of the force and the minimization of unwanted forces (such as those due to air currents). Because the arguments for double-arm, single-pan and electronic balances are a little different, the three are presented in sequence.

\section{a. The Double-Arm Balance (Double-Pan Balance)}


In concept, the traditional double-arm balance brings the forces on the two pans to exact equality. Under this condition the forces acting on the weight(s) or material placed un one pan equal the forces acting on the weight(s) or material placed on the other pan. The equation for this balance is developed as follows:

$$
\sum_{i=1}^{n_{1}} m(1, i) g,[1-\rho / d(1, i)]=\sum_{j=1}^{n_{2}} m(2, j) g[1-\rho / d(2, j)]
$$

where $m(1, i)$ is the true mass of weight $i$ on pan 1 which has an associated density of $d(1, i), g$ is the acceleration due to gravity, $\rho$ is the density of air (or fluid); and $m(2, j)$ is the true mass of weight $j$ on pan 2 which has an associated density of $d(2, j)$. Complexity is the result of two sets of weights, one of which may be a material whose weight is being determined. If all weight(s) have the same density the equation becomes much simpler as the buoyant force fraction $(1-p / d)$ is the same on both sides of the equation and cancels. The acceleration due to gravity cancels from the equation in a11 cases. The load on each pan varies as the mass on the pan increases, leading to some peculiar behavior for the double-arm balance. This peculiar behavior occurs as the result of change in the loading of the knife edges supporting the beam and the pans as weight on the pans is changed. A multiplication of force is possible if the arms of the balance are of unequal length; however, the usual form in precision balances has equal-arms.

b. The Single-Pan Balance

The single-pan balance is actually a double-arm balance, often with unequal arms, but with each loaded to the capacity of the balance for any weight placed on the pan. To accomplish this, the weighing arm is loaded internally by the full range of removable weights. In operation, the weights of the internal set are removed to compensate additional weight or weights added to the pan. Because the load on the knives is constant, balance behavior is more uniform. However, the balance equation is exactly the same as that for the double-arm balance described above [Equation (5)] but the reference weights $m(1, i)]$ and associated densities $[d(1, i)]$ are those of the internal weight set.

The operation of a single-pan balance is similar to a double-pan balance used in the substitution mode, where one pan is loaded with a tare weight. The zero'condition of the single-pan balance is at full load on the beam. Removal of weights from the weighing arm removes the net force on these weights (force due to gravity - buoyant force) from the weighing arm but exactly this force is left on the tare arm in effect. This is replaced by the load being weighed (force due to 
gravity - buoyant force) to re-establish the balanced condition and the load on the beam.

The single-pan balance is direct reading because it contains a full weight set and a means of indicating which weights have been removed from the weighing arm. Such balances are often more convenient to use than balances which require external weights. However, calibration of the internal weights is complicated because they cannot practically be removed and because of limited resolution of the balance in comparison with external standards.

\section{c. The Electronic Balance}

Electronic balances are, most often, devices which respond to force but which are provided with an electronic readout scaled directly in mass units. As force measuring devices they are affected by changes in the acceleration due to gravity and cannot be moved from one location to another significantly different without being recalibrated against reference weights. Because they must be adjusted against reference weights an equation similar to (5) above applies:

$$
\sum_{i=1}^{n_{1}} m(1, i) g[1-\rho(1) / d(1, i)]=\sum_{j=1}^{n_{2}} m(2, j) g[1-\rho(2) / d(2, j)]
$$

where terms are as in Equation (5) above except $p(1$ ) is the air density at the time the balance is calibrated and $p(2)$ is the air density at the time a weighing of mass set 2 is done. This assumes that all other factors (drift of electronic sensor and readout as well as mechanical stability of the balance) remain constant. As is the case for other balances, the acceleration due to gravity drops out subject to the additional constraint that the balance is not moved to a location with a gravity constant different from that at the calibration site.

Not covered specifically in the discussion are hybrid balances which are formed in part as a single pan mechanical balance and in part as an electronic balance. Because built-in weights are employed, the buoyant effect is as described above under the single-pan balance. Operation of the hybrid balance is otherwise similar to that of the electronic balance. In general, Equation (5) applies to these balances but an understanding of the operation of the particular balance is required to achieve best results.

As has been noted in the description of the three balance types, weighing is not dependent on the gravity constant (provided the constraint on electronic balances is met). Buoyancy, however, does affect the result. This effect is smaller than that for force, in general, because it amounts 
to the difference between the buoyant effect for standard and unknown weights (or material). An additional problem in the weighing of unknown material is that the density (or volume) is often not known, a situation which makes correction for the buoyant effect not possible. Values of the difference are given for typical materials in the table below, all calculated against reference weights with density of 8.0 grams/cc:

\section{TABLE II}

BUOYANCY OFFSET IN PARTS PER MILLION AT:

\begin{tabular}{|c|c|c|}
\hline $\begin{array}{l}\text { DENSITY } \\
\text { (grams/cc) }\end{array}$ & $\begin{array}{l}\text { SEA LEVEL } \\
(p=1.2 \\
m i 11 \text { igrams } / \mathrm{cc})\end{array}$ & $\begin{array}{l}1 \text { MILE ALTITUDE } \\
(p=0.986 \\
\text { miliigrams } / c c)\end{array}$ \\
\hline 2.7 (Aluminun) & 294.6 & 242.0 \\
\hline 7.7 & 5.8 & 4.8 \\
\hline 8.4 (Brass) & 7.1 & 5.9 \\
\hline 16.6 (Tantalum) & 77.7 & 63.9 \\
\hline 18.5 (Tungsten) & 85.1 & 70.0 \\
\hline
\end{tabular}

Notes: 1. Density 7.7 grams/cc is typical of internal weights in some older single-pan Balances. Newer balances often contain weights with densities approximating 8.0 grams/cc.

2. The range of 2.7 grams/cc to $1.8 .5 \mathrm{grams} / \mathrm{cc}$ approximates the range of densities found in rormal weighing. Resolution of many balances far exceeds the error of neglected buoyancy correction.

3. Sign of the offset is not given.

4. Density 8.4 is representative not only of Brass but also of some Nickel-Chromium which has been used for sheet-metal weights. In the past Tantalum has also been used for sheet-metal weights up to 1 gram.

\section{TOPIC 3. "APPARENT MASS"}

The process of weighing is complicated by the fact that the forces on two weights are actually being brought to balance, but the balances and weights used are calibrated in Mass units. As noted above, if the reference and unknown weights have the same density (same volume), the forces in balance "appear" to indicate that the Masses of the two are equal. However, this same density condition can only rarely be met, if ever. often, the material being weighed does not have a known density. This has led to the practice of weighing in terms of an "Apparent Mass" relative to an 
arbitrary density reference weight and set of conditions which determine the "reference" material. Historically, Brass with density 8.4 grams/ce at 0 Degrees Celsius and Sea Level was the reference for "Apparent Mass Versus Brass." This fiction has been convenient, but the reference conditions do not represent practical laboratory conditions.

More recent practice has used an arbitrary density of 8.0 grans/cc at the reference conditions of 20 Degrees Celsius and Sea Level. While somewhat more representative of conditions in weighing, the practice is not an acceptable substitute for actual conditions where accurate weighing must be made. Additionally, the use of "Apparent Mass" tends to hide the need for buoyancy corrections and the error evoked by failure to do so.

The lack of knowledge concerning the internal weights of a balance can be avoided by using the balance as a comparator in which weighing of both a reference and an unknown weight is sequentially done. More enhanced versions of this technique use inultipla weighings, generally in a fixed pattern of operations, and include addition of "sensitivity" weights at appropriate steps to calibrate the balance. The fixed pattern permits statistical analysis of the data to determine the uncertainty of each weighing. The mathematics of this process and the nature of the patterns required are beyond the subject for this document. Such techniques result in significantly more precise weighing but mandate the use of computer analysis of the data and require an even more precise knowledge of the density of the weights employed and correction for the effects of buoyancy.

\section{THE NEW STANDARD}

In 1978 a new and different standard was adopted in the United States detailing the specifications for mass standards. This new standard is in harmony with the standards adopted throughout the world and is available from ASTM. ANSI or from the Office of International Standards, Room A413, Admin., National Institute of Standards and Technology or NIST (formerly the National Bureau of Standards or NBS), Gaithersburg, MI 20899. The new Standard is designated ANSI/ASTM E617-78 "Standard Specification for LABORATORY WEIGHTS AND PRECISION MASS STANDARDS". It functionally replaces NBS Circular 547, Section 1, "PRECISION LABORATORY STANDARDS OF MASS AND IABORATORY WEIGHTS" and initiates many changes in the method by which weights are specified.

Slow to gain use by some manufacturers, the provisions of the new standard are now being seen in many specifications; however, both methods of specification may still be seen. The large variety of descriptors for weights resulting from this practice has led to considerable confusion. Problems have been experienced in ordering new weights because of changes in range of allowed densities under the new standard and in the change of "apparent mass" reference density and i.ts conditions. Problems have also been experienced because of the different methods of specifying weights in the two standards. The new standard deals more directly with the buoyancy problem and practices at the NIS'T and at other 
laboratories involved in high-precision weighing.' To present the changes more completely, two topics must be considered:

\section{APPARENT MASS REFERENCE STANDARD.}

The basic logic behind the use of "Apparent Mass" is given above. The use of "Apparent Mass" can be helpful in checking of balances and in normal everyday weighing particularly where the density of the unknown is truly unknown.

Historically, the choice for "Apparent Mass" was brass; most weights were made of brass. Another sign of historic time was the choice of "standard reference conditions"; i.e., 0 Degrees Celsius for temperature and Sea Level for pressure, which corresponded to prevalling engineering practice. Unfortunately, the reference conditions do not correspond well to the conditions met in most weighing. Finally, brass is not a good reference material as it is subject to corrosion. Protection of the brass by organic coating or by plating is not entirely satisfactory for high-precision weights although such weights are adequate for lower-precision weighing. Accordingly, preference in precision weights has been towards alloys which are highly corrosion resistant, particularly the stainless steels which have densities in the range 7.7 to 8.1 grams/cc compared to brass at about 8.4 grams/cc.

As adopted by the new standard, today's choice for an "Apparent Mass" reference is of density 8.0 grams/cc at 20 Degrees Celsius and in an air density of 1.2 milligrams/cc (sea level). This choice of reference is closer to weighing conditions and the density of today's preferred weights. It is a better compromise than the earlier practice, but it is still a compromise. Newer balances are made with internal weight sets which approximate the 8.0 grams/cc density and comply with the new standard. Older balances which have weights of: density other than 8.0 may be more precise or otherwise identical to newer balances; there is no cause to rush to replacement.

\section{DESIGNATION OF NEIGHTS.}

In NBS Circular 547, weights were described as belonging to Class J, $M, S, S-1, P, Q$ or $T$ with limits specified for Material, Design, Tolerance, etc. The weights most often of interest were those of Class $M, S$, or $S-1$. Class $M$ weights $z$ re one-piece and can have individual densities determined while Ciasses $S$ and $S-1$ are two-piece and density mist be assumed.

In the new standard different designations are used:

a. Type: Type 1 weights are of one-piece and Type 2 weights are of two-piece construction. 
Page 9

b. Grade: S, O, P, and Q describe a set of design limitations including range of permitted density, hardness; surface finish, corrosion resistance, etc.

c. Class: Classes $1,2,3,4,5$, and 6 are tolerance limitations with smaller numerical numbers representing tighter tolerances.

In general, weights described by the terminology of the older standard can also be described by the terminology of the new standard. A problem may occur where the allowed density in the older is larger than that in the new, but this causes no problem where buoyancy is corrected for and the density is known.

\section{PRIMARY STANDARDS LABORATORY PRACTICE.}

The Primary Standards Laboratory (PSL) follows the terminology of the new standard (ANSI/ASTM E617-78) on all weights calibrated. This practice does not: necessarily mean that a welght meets all requirements of the new standard, particularly as regards apparent offset from nominal (tolerance) and density. For Standards Laboratory weights the value is more important than a go/no-go indication the weight is within a particular tolerance limit. Equaliy, density of the particular weight is also reported rather than simply that it lies within an accepted range.

The PSL reports the True Mass of each weight in a set and either a measured density or an assumed density (as in the case of Type 2 weights with 2-piece construction and adfustment material). The assumed density is based on manufacturers data or published data for the particular material of which the weight is made. All Type 1 high quality weights have had their density determined by hydrostatic weighing during the first calibration (except for the sheet-metal weights).

Only the True Mass is normally given; however, "Apparent Mass versus Density 8.0 grams/cc". is given in addition to True Mass on request. Special weighing and reporting of mass values can be arranged on an as needed basis. Because of the press of calibration of Mass Standards and weights for Dead-Weight Pressure Apparatus, "lower priority is assigned to such measurements.

\section{E. RECOMMENDED PRACTICE FOR STANDARDS LABORATORIES}

The following practices are recomnended for standards Laboratories:

1. True Mass is required on all weights for use in weighings that will be duplicated elsewhere. Therefore, it is recommended that True Mass be given for all precision weights. The density used for each weight should also be given with a note when it has been assumed.

2. "Apparent Mass" should be given only using Density 8.0 grams/cc to 
harmonize practice with the current standard. As discussed above "Apparent Mass Versus Brass (density $8.4 \mathrm{grams} / \mathrm{cc}$ )" is now archaic and is principally useful only in comparing current with historical values on older weights.

3. Strongly recommended is the use of "Apparent Mass versus Density 8.0 grams/cc At Representative (Average) Local Conditions" especially for weigh.s used in checking precision balances. The advantage of this practice is that the values for the weights can be used directly in checking high-precision balances or as reference for electronic balances without computation. The average of local conditions is generally adequate as the variation from these conditions is relatively small in all but unusual circumstance. Weight materials are not avallable with exactly 8.0 grams/cc density but this practice gives the corrected or "Apparent Mass" value as though they have that density. Accordingly, such values can be used without calculation for calibration of low and moderate precision balances and as standards for moderate precision weighing.

4. Strongly recommended is the practice of considering the weights the standard wherever possible (rather than the balance). This is the correct approach because, as noted above, weights in a balance cannot be determined better than the resolution of the balance while they are in place and cannot practically be removed for separate calibration. In a similar manner, resolution and repeatability of. electronic balances limit achievable uncertainty.

5. The oft-repeated request for "better weights" is difficult. If closer to nominal is desired it should be noted that weights not conforming to the standard incur a significant surcharge in procurement and because of buoyancy problems may be less than desired in use. Material with a density of exactly 8.0 grams/cc is unavailable; however, the properties of the non-magnetic stainless steels. (density 7.7 to 8.1 grams/cc) make them almost perfect weight materials. It is inescapable that those desiring to perfcrm precison weighing must consider the effects of buoyancy, both on their reference standard weights and on the material being weighed. Failure to do so is a failure to weigh. 
Page 1, Appendix

\section{APPENDIX A: DETERMINATION OF BUOYANCY CORRECTIONS}

Use of the balance equation for single masses is the simplest way to develop the equations necessary for computing "Apparent Mass". Equation (5). in the text, rewritten for this condition, is:

$$
m(1)[1-\rho(1) / d(1)]=m(2)[1-\rho(2) / d(2)] ;
$$

where $\mathrm{m}(1)$ is the true mass on the left side which has an associated density $d(1)$ and is buoyed up by fluid of density $\rho(1)$, and $m(2)$ is the true mass on the right side, which has an associated density of $\mathrm{d}(2)$ and is buoyed up by fluid of density $\rho(2)$.

For single masses, Equation (7) brings to balance the ForCEs acting on the two weights $m(1)$ and $m(2)$. For use in air where $\rho(1)$ and $\rho(2)$ are the same pressure, the "Apparent Mass at Density d(1)" may be the left side while the right has the T'RUE MASS at the actual density. Note that the "Apparent Mass" is exactly the value of an equivalent TRUE MASS but at density $d(1)$ to produce the same balancing force. It is this "fictitious" "Apparent Mass" that causes much misunderstanding even though the concept is relatively simple.

An additional use of Equation (7) is in the deternination of the density of a weight by hydrostatic weighing; a method for so doing $i$ a given in APPENDIX $B$.

other forms of Equation (7) are often seen in the literature. Common forms are:

$$
\begin{aligned}
& m(1)=m(2)\left(\frac{1-\rho(2) / d(2)}{1-\rho(1) / d(1)}\right) \text {, and } \\
& m(1)=m(2)-\rho(2) v(2)+\rho(1) v(1)
\end{aligned}
$$

where terms are as in Equation (7) above or d has been replaced by $\mathrm{m} / \mathrm{v}$ (mass divided by volume).

Equations (8) and (9) are both exact. Note that d (density) is a basic property of the material of which the weight is made while $v$ (volume) is a measure of how much of the material. with density $d$ is in the weight. Should a piece of the weight be removed, $v$ will change but d will not. For precision weights that are not roughly handled the choice of which equation to use is at the convenience of the weighing technician.

A third form of the equation for the case where $\rho(1)=\rho(2)=\rho$ may also be seen. This equation is an approximation based on the observation that $\rho / d$ is sma11 compared to 1 :

$$
m(1)=m(2)\left[1-\rho\left(\frac{1}{d(2)} \cdot \frac{1}{d(1)}\right)\right] .
$$


Using this approximate equation for any $d(2)$ greater than 2 and for $d(1)$ with value 8.0, the error is no greater than 1 part in a milition. This result is sufficiently precise for many weighing operations and, before the advent of calculators, this was often the equation of choice because of simplicity.

\section{EXAMPLES :}

A. Equation (8), was used to compute the "Apparent Masses" and, from them, the BUOYANCY OFFSETS 1isted in Table IT of the text. The weight for which the "Apparent Mass" values were computed was assumed to have a unity TRUE MASS value $(\operatorname{m}(2)=1.00)$, to have the densities listed in Table II, and to be either at sea level (air density $=0.0012$ grams/cc) or at the PSL (air density $=0.000986$ grams/cc). Rather than the whole factor, only the difference from unity is given by use of:

$$
\text { BUOYANCY OFFSET (in ppm) }=1,000,000[\mathrm{~m}(1)-1] \text {. }
$$

As noted, the sign is not given in the table.

B. Examples of converting a value of apparent mass versus normal brass to apparent mass versus density 8.0 at local conditions using weight density and volune are as follows:

Given: A weight of nomal brass (nominal. 100 grams) is adjusted to a true mass of exactly 100.00000 grams. Normal brass has a density of 8.4 grams/cc at 0 degrees Celsius ( 8.3909 grams/cc at 20 degrees Celsius). The air density at the location of the weight is 0.000987 grams/ce; the temperature is 20 degrees Colsius.

Problem: Determine the apparent mass versus density 8.0 at local conditions using a11 three equations (in order (8), (10), and (9)).

Note: Apparent mass versus normal brass is defined at 0 degrees Celsius and sea level density air (0.0012 grams/cc)'.

Apparent mass versus density 8.0 is defined at 20 degrees Celsius and sea level air density.

This problem is a bit different as apparent mass versus density 8.0 at local conditions is specified.

Solution using exact equation (8):

$$
\begin{aligned}
\text { Apparent mass } & =100.00000\left(\frac{1-0.000987 / 8.3909}{1-0.000987 / 8.0}\right) \\
& =100.00057[4828] \text { grams. }
\end{aligned}
$$

Solution using approximate equation (10):

$$
\text { Apparent: } \text { mass }=100.00000[1-0.000987(1 / 8.3909-1 / 8.0)]
$$




$$
=100.00057[4757] \text { grams. }
$$

Note that the difference between the exact and approximate equations occurs in figures that are below 1 part in 10 million.

Solution using exact equation (9):

Equation (9) requires the apparent volume of the weight be known both for density 8.3909 and for density 8.0. However, in advance of solution, the apparent mass at density 8.0 is not known. Therefore, the solution will employ an iterative process the first step of which is to compute an approximate volume under density 8.0 conditions.

Approximate volume (initial value) $=100.00000 / 8.0=12.5 \mathrm{cc}$.

1st apparent mass $=100.00000-0.000987(100 / 8.3909-12.5)$

$$
=100.00057[4757] \text { grams } .
$$

While the result is certainly close enough for most purposes it is not yet exact. A new volume is computed:

Approximate volume (second value) $=100.000574757 / 8.0$

$$
=12.5000718446 \mathrm{cc} \text {. }
$$

2nd apparent mass $=100.00000$

$$
0.000987(100 / 8.3909-12.5000718446)
$$

$$
=100.00057[4828] \text { grams. }
$$

The result is now in agreement with that of equation (8). Only one iteration was required in the computation because of the relatively small difference between the two volumes. The final volume is computed as follows:

Apparent volume (final value) $=100.000574828 / 8.0$

$$
=12.5000[718535] \mathrm{cc} \text {. }
$$

The final value for the apparent volume will not result in a further change in the apparent mass. If a larger difference between initial and final volumes must be accommodated, more iterations may be necessary. It also should be noted that more figures than necessary have been carried along in the volume value, a difficulty caused by "believing" the computer readout. It is most appropriate to round the figure back to no more than 4 places past the decimal point. The apparent volume is known no better than the density of the weight. The density of very high quality weights is not known to better than / significant tigures; 
Page 4, Appendix

the densities of lesser weights must often be estimated from manufacturers data (2 piece weights).

C. An example of computing the difference in "apparent mass under local conditions" for weights which have been adjusted to "apparent mass versus brass" but which have an actual density other than brass $(8.4 \mathrm{~g} / \mathrm{cc})$ or $8.0 \mathrm{~g} / \mathrm{cc}$. This example is of a very practical problem. Older single-pan balances were made with brass weights which had densities approximating $8.4 \mathrm{~g} / \mathrm{cc}$. Recent singlepan balances have weights made of material which has density approximating 8.0 $\mathrm{g} / \mathrm{ccc}$. Many balances of moderate age were made with weight material having 7.77 $\mathrm{g} / \mathrm{cc}$ density but the weights were trimmed to "apparent mass versus brass." The offset must be accounted for if the continued use of these balances is warranted; it is noted that these older balances are often more precise than the newer electronic balances which cover the same range. It should also be noted that buoyancy correction is required even when "apparent mass versus brass" is used but the correction is much simpler. Note finally that at $20^{\circ} \mathrm{C}$ density 8.4 is 8.3909 (the Normal Brass historically used had a defined expansion coefficient).

Given: A single pan balance which has internal weights of density $7.77 \mathrm{~g} / \mathrm{cc}$ adjusted to exact nominal mass values in "apparent mass versus brass."

Problem: The balance is used at one mile altitude (air density $0.000986 \mathrm{~g} / \mathrm{cc}$ ) compared with the sea level air density of $0.0012 \mathrm{~g} / \mathrm{cc}$. Also, as the calibrating weights are of density $8.0 \mathrm{~g} / \mathrm{cc}$, the "apparent mass versus density 8.0 under local conditions" is required. It is presumed that all measurements are made in $20^{\circ} \mathrm{C}$.

Solution is obtained by a double application of exact equation (8). The first application is to obtain the TRUE MASS of a weight versus its own density under adjustment conditions (the "apparent mass versus brass" value is assumed to be 1.00). The second application is to obtain the "apparent mass" under the actual use conditions. Because a unity value is assumed this approach gives the correction factor for a weight of any value.

$$
(\text { TRUE MASS })\left(1-\frac{0.0012}{7.77}\right)=(1.0000 \cdots)\left(1-\frac{0.0012}{8.3909}\right)
$$

As noted above, this equation is used to obtain (TRUE MASS) of the weights. A second application of (8) yields the ("apparent mass") under the test conditions.

$$
\text { (APPARENT MASS) }\left(1-\frac{0.000986}{8.0}\right)=(\text { TRUE MASS })\left(1-\frac{0.000986}{7.77}\right)
$$

Combining these two equations yields: 


$$
\begin{aligned}
(\text { APPARENT MASS }) & =(1.0000 \ldots)\left[\frac{\left(1-\frac{0.0012}{8.3909}\right)\left[1-\frac{0.000986}{7.77}\right]}{\left[1-\frac{0.0012}{7.77}\right)\left(1-\frac{0.000986}{8.0}\right]}\right] \\
& =(1.000 \ldots)(1+7.7[8102] \mathrm{ppm})
\end{aligned}
$$

In this result, the figures in the brackets are questionable. However, it is necessary that the $7.8 \mathrm{ppm}$ difference be expected for all weights in such a balance.

\section{APPENDIX B. A METHOD FOR MEASURING AND CALCUIAATING DENSITY}

It is often desirable to determine the density of an unknown weight or of a solid material. The method described is useful in this determination; three weighings are required:

1. A determination of the apparent mass in air: Correction is made for the reference weight buoyancy but not for the unknown as follows:

$$
M(a)=\sum_{i=1}^{n_{1}} \ln (i)[1-\rho(a) / d(i)],
$$

where $M(a)$ is the apparent mass of the unknown in air, $m(i)$ is one of the weights required to balance the unknown having density $d(i)$ and $\rho(a)$ is the air density. Note that $\rho(a)$ will be required later in computing both density and true mass of the unknown.

2. A determination of the apparent mass of the unknown in water (or other fluid with known density) plus the tare weight of the hanger (part in water and part in air): Correction is made for the reference weight buoyancy as follows:

$$
M(w 1)=\sum_{j=1}^{n_{2}} m(j)[1-\rho(a) / d(j)],
$$

where $M(w 1)$ is the apparent mass of the unknown plus the hanger, $m(j)$ and $d(j)$ are the weights and their associated densities required for balance, and $\rho(a)$ is again air density. Note that $\rho(w)$, the water or immersion fluid density (at the 
temperature during the weighing) will be required later in computing both density and true mass of the unknown. It is necessary to know both $\rho(a)$ and $\rho(w)$ at the temperature of measurement.

3. A determination of the apparent mass of the hanger under the conditions of 2 . above: This is accomplished by dislodging the unknown from the hanger but leaving it in the water so that the depth of immersion of the hanger will be essentially identical with that of 2.; correction is made for buoyancy of the balancing weights:

$$
M(w 2)=\sum_{k=1}^{n_{3}} m(k)[1-\rho(a) / d(k)],
$$

where $M(w 2)$ is the apparent mass of the hanger (or tare weight), $m(k)$ and $d(k)$ are the mass and density of the weights required for balance, and $\rho(a)$ is the air density.

The two apparent mass values $M(w 1)$ and $M(w 2)$ are first combined to produce the apparent mass, $M(w)$, of the unknown in water; then using the two apparent inasses, the density of the air and the density of the water, a direct solution for both the density, D, and true mass, $M$, of the unknown is obtained as follows :

$$
\begin{aligned}
& M(w)=M(w 1)-M(w 2), \\
& D=\frac{\rho(w) M(a)-\rho(a) M(w)}{M(a)-M(w)}, \text { and } \\
& M=\frac{\rho(w) M(a)-\rho(a) M(w)}{\rho(w)-\rho(a)} .
\end{aligned}
$$

The results may be easily verified by using equation (8). The uncertainty, however, is dependent on the balance used and the weighing process employed. One way of improving the uncertainty is to use the average of a series of weighings for each of the terms, $M(a), M(w 1)$, and $M(w 2)$. The uncertainty is obtained by an error analysis of the process. 

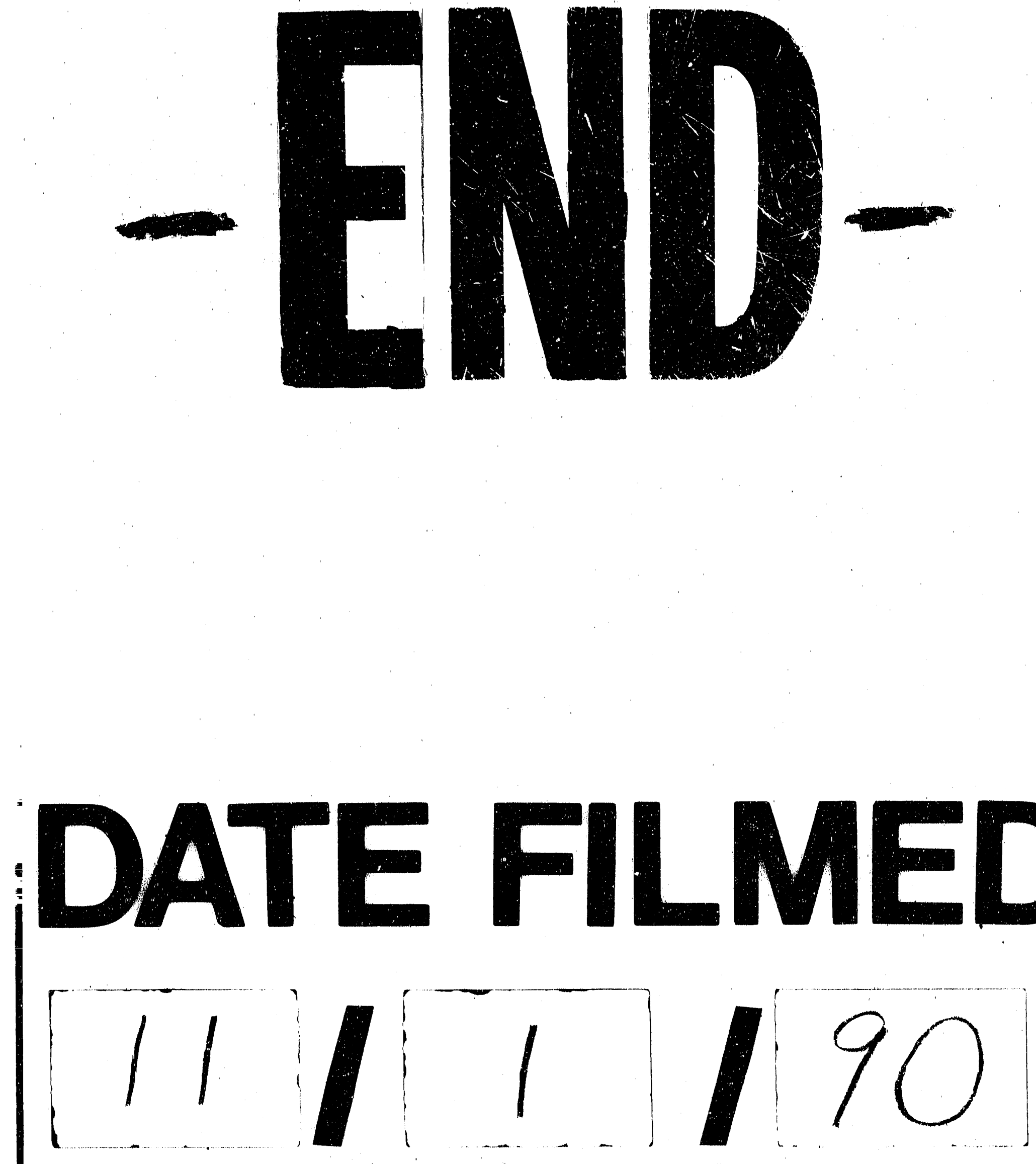
